\title{
Níveis de fósforo digestível para suínos em fase de crescimento
}

\author{
Silvano Bünzen ${ }^{1}$, Horacio Santiago Rostagno², Charles Kiefer ${ }^{3}$, Alexandre de Oliveira \\ Teixeira ${ }^{4}$, Valdir Ribeiro Junior ${ }^{1}$
}

\footnotetext{
1 Programa de Pós-Graduação em Zootecnia da Universidade Federal de Viçosa.

2 Universidade Federal de Viçosa.

3 Universidade Federal de Mato Grosso do Sul.

${ }^{4}$ Universidade Federal de São João del-Rei.
}

RESUMO - Este estudo foi conduzido com o objetivo de determinar a exigência de fósforo digestível de suínos em crescimento. Foram utilizados 70 suínos mestiços (35 machos castrados e 35 fêmeas), com peso médio inicial de 29,72 $\pm 1,52 \mathrm{~kg}$, distribuídos em delineamento de blocos casualizados, com cinco tratamentos, sete repetições e dois animais (um macho e uma fêmea) por unidade experimental. O experimento teve duração de 21 dias. Os tratamentos consistiram de rações à base de milho e farelo de soja suplementadas com fosfato bicálcico, correspondendo a cinco níveis de fósforo digestível (0,19;0,25; 0,30; 0,35 e 0,40\%). Para cada nível de fósforo estudado, foi adicionado calcário buscando manter constante a relação cálcio:fósforo das dietas. O aumento dos níveis de fósforo digestível influenciou de maneira quadrática o consumo de ração e o ganho de peso, cujos valores máximos foram obtidos nos níveis de 0,32 e $0,31 \%$ de fósforo digestível, respectivamente. Não houve efeito dos níveis de fósforo digestível sobre a conversão alimentar. Os teores de cinzas e de cálcio analisados nos metatarsos não foram influenciados pelos níveis de fósforo digestível, enquanto o teor de fósforo apresentou resposta linear. A exigência de fósforo digestível para suínos alimentados com dietas à base de milho e farelo de soja é de $0,31 \%$, que corresponde ao consumo diário de $5,87 \mathrm{~g}$ de fósforo digestível.

Palavras-chave: cálcio e fósforo, digestibilidade verdadeira, exigência

\section{Digestible phosphorus levels for growing swine}

\begin{abstract}
This study was carried out to determine the digestible phosphorus requirement for growing swine. Seventy crossbred swine (35 castrated males and 35 females), with initial weight of $29.72 \pm 1.52 \mathrm{~kg}$ were distributed in a completely randomized design with five treatments, seven replicates and two animals (one male and one female) per experimental unit. The experiment lasted 21 days. The treatments were composed of diets formulated with corn and soybean meal, supplemented with dicalcium phosphate, corresponding to five levels of digestible phosphorus $(0.19 ; 0.25 ; 0.30 ; 0.35$ and $0.40 \%)$. For each phosphorus level, limestone was added to maintain the calcium:phosphorus ratio constant. The increase in digestible phosphorus influenced feed intake and weigh gain quadratically, and the maximum values for those parameters were obtained with the levels of 0.32 and $0.31 \%$ digestible phosphorus, respectively. There was no effect of digestible phosphorus levels on the feed:gain ratio. The ashes and calcium contents analyzed in the metatarsi were not influenced by digestible phosphorus levels, while the phosphorus content in bone showed linear response. The requirement of digestible phosphorus for pigs fed diets based on corn and soybean meal is $0.31 \%$, corresponding to a daily intake of $5.87 \mathrm{~g}$ digestible phosphorus.
\end{abstract}

Key Words: calcium and phosphorus, requirements, true digestibility

\section{Introdução}

As exigências de fósforo de suínos têm sido determinadas por meio de valores de biodisponibilidade do fósforo nos alimentos com base na retenção desse mineral nos tecidos, em estudos comparativos com uma fonte de fósforo considerado como padrão 100\% disponível. Muitos desses estudos, no entanto, foram realizados na década de 90 e podem não ser representativos dos alimentos e animais contemporâneos, ambos submetidos ao constante melhoramento genético em busca da máxima eficiência de produção.

Tem-se constatado que dietas baseadas em fósforo disponível possuem normalmente excesso de fósforo por serem determinados por ensaios de retenção e promovem a excreção excessiva desse mineral nos dejetos (O’Quinn et al., 1997), com prejuízos econômicos e ao meio ambiente, já que o fósforo é considerado o terceiro nutriente mais oneroso de rações para monogástricos (Jongbloed \& Kemme, 1990). 
Para estimar a excreção de fósforo por meio dos dejetos, é necessário medir a digestibilidade do fósforo nos alimentos. Quando esses dados são mensurados, pode-se estimar a quantidade de fósforo absorvido pelo trato gastrintestinal, reduzindo as quantidades excretadas (Stein et al., 2008). Por sua vez, as exigências de fósforo dos animais podem ser determinadas em ensaios de disponibilidade e digestibilidade, com a vantagem que a análise de digestibilidade ser um ensaio que demanda menor custo e tempo, além de permitir a formulação de dietas buscando a mínima excreção de fósforo (O’Quinn et al., 1997).

Diversos pesquisadores têm sugerido que os coeficientes de digestibilidade do fósforo podem ser utilizados para avaliar o aproveitamento do fósforo pelos suínos (Jongbloed \& Kemme, 1990; Fan et al., 2001; Shen et al., 2002) e, dessa forma, formulações mais precisas poderiam contemplar níveis de fósforo nas dietas mais próximas as exigências dos animais, reduzindo custos e a excreção desse mineral. Os coeficientes de digestibilidade aparente do fósforo, por sua vez, subestimam o real aproveitamento do fósforo pelos animais por não contemplar as perdas de fósforo endógeno, que ocorrem por meio de muco, descamações e enzimas (Shen et al., 2002).

Diversos estudos têm sido conduzidos para determinação dos valores de fósforo digestível dos diferentes alimentos e, assim, tornar possível a formulação de dietas com níveis de fósforo mais próximos às exigências nutricionais dos suínos e considerando o seu real aproveitamento nos alimentos (Bohlke et al., 2005; Widmer et al., 2007; Bünzen et al., 2008), reduzindo custos com a suplementação inorgânica e a excreção deste mineral.

Neste contexto, este trabalho foi realizado com o objetivo de determinar as exigências de fósforo digestível para suínos dos 30 aos $50 \mathrm{~kg}$ alimentados com dietas à base de milho e farelo de soja.

\section{Material e Métodos}

O experimento foi conduzido no setor de Suinocultura do Departamento de Zootecnia do Centro de Ciências Agrárias da Universidade Federal de Viçosa, em Viçosa,
Minas Gerais. Foram utilizados 70 leitões híbridos comerciais, machos castrados e fêmeas, com peso médio inicial de 29,72 $\pm 1,52$ kg durante período experimental de 21 dias.

Os animais foram alojados em baias com piso de concreto, dotadas de comedouros semiautomáticos e bebedouros tipo chupeta, localizadas em prédio de alvenaria coberto com telhas de cerâmica.

Os animais foram distribuídos em delineamento experimental de blocos ao acaso, com cinco níveis de fósforo digestível, sete repetições e dois animais por unidade experimental, sendo um de cada sexo. A unidade experimental foi representada pela baia. Na formação dos blocos, foram considerados o peso inicial e o parentesco dos animais.

A dieta-referência foi formulada à base de milho e farelo de soja, de acordo com as exigências nutricionais dos suínos, seguindo as recomendações de Rostagno et al. (2005). A concentração de fósforo digestível dos alimentos utilizados para elaboração das dietas experimentais (Tabela 1) foi baseada nos valores estabelecidos por Bünzen (2010) para suínos em fase de crescimento.

A partir da dieta-referência, realizou-se a suplementação de fosfato bicálcico para se obterem as dietas com os cinco níveis de fósforo digestível $(0,19 ; 0,25 ; 0,30 ; 0,35$ e 0,40\%). Para cada nível de fósforo estudado, adicionou-se calcário à dieta como fonte de cálcio buscando manter constante a relação cálcio:fósforo (Tabela 2).

Os animais receberam as dietas experimentais e água à vontade. Foram realizadas pesagens periódicas das sobras de ração e os animais foram pesados por ocasião ao início e término do período experimental para determinação do ganho de peso, consumo de ração e conversão alimentar.

Ao final do período experimental, após a pesagem, foi abatido um animal de cada unidade experimental, sendo escolhido o animal com peso mais próximo da média do bloco, para coleta do osso metatarso do membro anterior direito.

Os ossos foram secos em estufa ventilada a $80{ }^{\circ} \mathrm{C}$, por 72 horas e, então, submetidos à retirada de gordura por extrator Soxhlet, por 4 horas, e colocados em mufla a $600^{\circ} \mathrm{C}$, durante 4 horas, para determinação de cinzas. Em seguida,

Tabela 1 - Conteúdo de fósforo total e digestível verdadeiro dos ingredientes utilizados nas dietas experimentais

\begin{tabular}{lcccc}
\hline Ingrediente & $\%$ P total ${ }^{1}$ & CDV P suínos ${ }^{2}$ & \% P digestível $^{2}$ & \% Cálcio $^{1}$ \\
\hline Milho & 0,24 & 53,19 & 0,13 & - \\
Farelo de soja & 0,53 & 48,51 & 0,26 & - \\
Fosfato bicálcico & 18,50 & 74,79 & 13,84 & 25,30 \\
Calcário & - & - & - & 33,52 \\
\hline
\end{tabular}

${ }^{1}$ Valores analisados.

${ }^{2}$ CDVP = coeficientes de digestibilidade verdadeira do fósforo (valores calculados). 
Tabela 2 - Composição percentual e calculada das dietas experimentais

\begin{tabular}{|c|c|c|c|c|c|}
\hline \multirow[t]{2}{*}{ Ingrediente } & \multicolumn{5}{|c|}{ Nível de fósforo digestível (\%) } \\
\hline & 0,19 & 0,25 & 0,30 & 0,35 & 0,40 \\
\hline Milho grão & 72,86 & 72,86 & 72,86 & 72,86 & 72,86 \\
\hline Óleo soja & 0,84 & 0,84 & 0,84 & 0,84 & 0,84 \\
\hline Sal comum & 0,39 & 0,39 & 0,39 & 0,39 & 0,39 \\
\hline Suplemento vitamínico & 0,13 & 0,13 & 0,13 & 0,13 & 0,13 \\
\hline L-treonina & 0,04 & 0,04 & 0,04 & 0,04 & 0,04 \\
\hline Butil-hidroxi-tolueno & 0,01 & 0,01 & 0,01 & 0,01 & 0,01 \\
\hline Fosfato bicálcico & 0,29 & 0,67 & 1,05 & 1,42 & 1,80 \\
\hline Calcário & 0,71 & 0,69 & 0,69 & 0,66 & 0,63 \\
\hline Inerte $^{1}$ & 1,50 & 1,14 & 0,76 & 0,41 & 0,07 \\
\hline Total & 100,00 & 100,00 & 100,00 & 100,00 & 100,00 \\
\hline Metionina + cistina digestível (\%) & 0,54 & 0,54 & 0,54 & 0,54 & 0,54 \\
\hline Treonina digestível (\%) & 0,58 & 0,58 & 0,58 & 0,58 & 0,58 \\
\hline Sódio (\%) & 0,18 & 0,18 & 0,18 & 0,18 & 0,18 \\
\hline Cálcio (\%) & 0,42 & 0,50 & 0,60 & 0,68 & 0,76 \\
\hline Fósforo total (\%) & 0,35 & 0,42 & 0,50 & 0,56 & 0,63 \\
\hline Fósforo digestível $(\%)^{2}$ & 0,19 & 0,25 & 0,30 & 0,35 & 0,40 \\
\hline
\end{tabular}

Dietas formuladas segundo recomendações de Rostagno et al. (2005).

${ }^{1}$ Areia lavada.

${ }^{2}$ Valores calculados com base nos coeficientes de digestibilidade (Tabela 1).

foram analisados os teores de fósforo e cálcio neles contidos (Silva \& Queiroz, 2002).

As análises bromatológicas dos ingredientes e das dietas experimentais foram realizadas de acordo com a técnica descrita por Silva \& Queiroz (2002), no Laboratório de Nutrição Animal do Departamento de Zootecnia, da Universidade Federal de Viçosa (UFV). A determinação do teor de cinza ósseas, de fósforo e de cálcio nos ossos foi realizada no Laboratório da Rodes Química Cajati LTDA, no município de Cajati, São Paulo.

As análises estatísticas foram realizadas utilizando-se o programa SISVAR (Ferreira, 2000), a 5\% de significância. As estimativas das exigências nutricionais de fósforo digestível foram feitas por meio de modelos de regressão polinomial, considerando-se na escolha do modelo, uma vez respeitada a interpretação biológica, o valor da menor soma de quadrados dos desvios.

\section{Resultados e Discussão}

O consumo total de ração no período e o consumo diário apresentaram efeito quadrático $(\mathrm{P}<0,03)$ em função dos níveis de fósforo digestível (Tabela 3), de acordo com a equação $\hat{Y}=25,25787+329,69246 x-497,29444 x^{2}, R^{2}=0,78$.

Entre os estudos realizados para estimar as exigências de fósforo digestível, estão o de Alebrante et al. (2011), que observaram variação quadrática do consumo de ração em decorrência da variação dos níveis de fósforo disponível entre 0,107 e $0,642 \%$. Por sua vez, Saraiva et al. (2009) verificaram aumento linear no consumo de ração quando testaram níveis de 0,11 a 0,54\% de fósforo disponível para suínos de 15 a $30 \mathrm{~kg}$. Os autores, no entanto, atribuíram este resultado ao baixo consumo na fase inicial, fato não evidenciado neste estudo, no qual o consumo obtido foi próximo ao preconizado por Rostagno et al. (2005) para

Tabela 3 - Desempenho de suínos em crescimento de acordo com os níveis de fósforo digestível

\begin{tabular}{|c|c|c|c|c|c|c|c|}
\hline \multirow[t]{2}{*}{ Variável } & \multicolumn{5}{|c|}{ Nível de fósforo digestível, \% } & \multirow[t]{2}{*}{$<\mathrm{P}$} & \multirow[t]{2}{*}{$\mathrm{CV}, \%$} \\
\hline & 0,19 & 0,25 & 0,30 & 0,35 & 0,40 & & \\
\hline Consumo de ração no período, kg* & 70,58 & 75,71 & 77,80 & 82,94 & 76,22 & 0,029 & 8,1 \\
\hline Consumo de ração diário, kg* & 1,68 & 1,80 & 1,85 & 1,97 & 1,81 & 0,029 & 8,1 \\
\hline Ganho de peso no período, kg** & 15,62 & 17,98 & 19,07 & 19,39 & 17,73 & 0,001 & 13,1 \\
\hline Ganho de peso diário, kg** & 0,745 & 0,856 & 0,908 & 0,925 & 0,858 & 0,001 & 12,6 \\
\hline Conversão alimentar & 2,27 & 2,10 & 2,04 & 2,15 & 2,15 & 0,239 & 7,2 \\
\hline
\end{tabular}

Efeito quadrático $*(\mathrm{P}<0,03) ; * *(\mathrm{P}<0,01)$. 
animais de desempenho médio. Por outro lado, O’Quinn et al. (1997) avaliaram a exigência de fósforo digestível para suínos em crescimento e terminação e não verificaram efeito sobre o consumo dos animais alimentados com dietas contendo diferentes níveis de fósforo digestível.

A variação do consumo de ração com o aumento dos níveis de fósforo observada nesse estudo pode ser um indicativo de que os suínos são sensíveis a níveis inadequados de fósforo na dieta, uma vez que a deficiência ou mesmo o excesso de fósforo afetam negativamente o consumo de alimento.

O ganho de peso diário sofreu efeito quadrático $(\mathrm{P}<0,01)$ dos diferentes níveis de fósforo digestível, o que difere dos resultados obtidos por O’Quinn et al. (1997), que não notaram efeito sobre este parâmetro. Stahly (2009) verificou efeito linear sobre o ganho de peso de suínos entre 9 e $37 \mathrm{~kg}$ de peso corporal e efeito quadrático para a fase posterior (37 a $65 \mathrm{~kg}$ ) ao avaliarem níveis de fósforo disponível variando entre 0,13 a $0,56 \%$.

A equação obtida para o parâmetro ganho de peso diário, no presente trabalho, foi $\hat{Y}=-0,152+6,663 x-10,32 x^{2}$, $\mathrm{R}^{2}=0,98$, obtendo-se o maior ganho de peso diário ao nível de $0,32 \%$ de fósforo digestível.

Por outro lado, os níveis de fósforo digestível não influenciaram $(\mathrm{P}>0,05)$ a conversão alimentar dos animais. Mesmo resultado foi obtido por O’Quin et al. (1997), que obtiveram a mesma resposta em suínos entre 20 e 50 kg, sendo o nível mais baixo ( $0,21 \%$ de fósforo digestível) sugerido pelos pesquisadores como o mais adequado para o melhor desempenho dos animais, uma vez que não houve efeito sobre a conversão e o ganho de peso. Os resultados desse estudo foram semelhantes aos descritos por Jongbloed \& Everts (1992), que obtiveram 0,23\% como a exigência de fósforo digestível para o melhor desempenho por também não terem observado efeitos sobre os parâmetros de desempenho avaliados.

Os resultados de exigência de fósforo podem variar consideravelmente entre as publicações, sendo que o principal fator dessa variação é o potencial genético dos animais que resulta em grande variação sobre a capacidade de deposição de carne magra na carcaça. Essa proposição foi confirmada em estudo realizado por Wiseman et al. (2007), que observaram que entre linhagens com grande capacidade de deposição de carne há diferenças.

Não foram constatados efeitos $(\mathrm{P}>0,05)$ dos níveis de fósforo digestível sobre o percentual de cinzas e cálcio nos ossos dos suínos (Tabela 4). Outros autores também não encontraram efeito sobre a porcentagem de cinzas de ossos de suínos alimentados com dietas contendo diferentes níveis de fósforo digestível (O’Quinn et al., 1997) e disponível (Hastad et al., 2004; Saraiva et al., 2009).

A porcentagem de fósforo nos ossos dos suínos foi influenciada $(\mathrm{P}<0,01)$ pelos níveis de fósforo digestível, aumentando de forma linear conforme o aumento do nível de fósforo da dieta, segundo a equação $\hat{Y}=3,848 x+7,471$, $\mathrm{R}^{2}=0$,88. Em geral, as exigências para máxima mineralização óssea são superiores em relação às exigências para o máximo desempenho dos animais (Ketaren et al., 1993; Gomes et al., 2004). Essa afirmação está de acordo com os resultados obtidos neste estudo, uma vez que para efeito de desempenho, representado pelo parâmetro ganho de peso, foi obtido resultado quadrático, evidenciando melhor ganho no nível de $0,32 \%$ de fósforo digestível, enquanto, para a deposição de fósforo nos ossos, os níveis estudados não foram suficientes para maximizar este parâmetro.

Neste estudo, o nível de 0,31\% de fósforo digestível calculado utilizando-se o intervalo de confiança de 95\% da quadrática (Tabela 5), de acordo com Sakomura \& Rostagno (2007), parece ser suficiente para atender o melhor ganho de peso de suínos em crescimento, embora a exigência para o percentual de fósforo nos ossos tenha ficado acima dessa estimativa.

Embora alguns estudos (Saraiva et al., 2009; Alebrante et al., 2010) tenham evidenciado a interdependência entre a deposição óssea de cálcio e fósforo, esse resultado não foi observado neste estudo, uma vez que o aumento do nível de fósforo nos ossos não foi acompanhado pelo aumento do percentual de cálcio. Provavelmente, o cálcio adicional pode ter sido complexado de alguma forma e que pode ter prejudicado sua absorção, porém não se mensurou a recuperação de cálcio e fósforo nas fezes, o que não esclarece totalmente a questão. Esses fatos dificultam a recomendação de níveis ótimos quanto a relação cálcio:fósforo digestível pela insuficiência de dados disponíveis sobre a

Tabela 4 - Parâmetros ósseos de suínos em crescimento de acordo com os níveis de fósforo digestível

\begin{tabular}{lccccccc}
\hline Variável & \multicolumn{3}{c}{ Nível de fósforo digestível, \% } & \multicolumn{2}{c}{ CV, \% } \\
\cline { 2 - 6 } & 0,19 & 0,25 & 0,30 & 0,35 & 0,40 & \\
\hline Cinzas (\%) & 45,23 & 48,37 & 47,78 & 47,91 & 48,04 & 0,194 \\
Fósforo (\%)* & 8,09 & 8,56 & 8,73 & 8,69 & 8,01 & 0,001 \\
Cálcio (\%) & 17,02 & 18,10 & 17,73 & 17,95 & 6,6 \\
\hline
\end{tabular}

* Efeito linear e quadrático $(\mathrm{P}<0,01)$. 
Tabela 5 - Exigência de fósforo digestível para suínos em crescimento estimados pelo modelo linear ou quadrático

\begin{tabular}{lccc}
\hline Parâmetro & Equação & Nível & $95 \%$ quadrática \\
\hline Consumo de ração diário, kg* & $\hat{Y}=25,25787+329,69246 x-497,29444 \mathrm{x}^{2}, \mathrm{R}^{2}=0,78$ & 0,33 & 0,32 \\
Ganho de peso diário, kg* & $\hat{Y}=-10,32 \mathrm{x}^{2}+6,663 \mathrm{x}-0,152, \mathrm{R}^{2}=0,98$ & 0,32 & - \\
Conversão alimentar & - & - & - \\
Cinzas, \% & $\hat{Y}=3,848 \mathrm{x}+7,471, \mathrm{R}^{2}=0,88$ & - & - \\
Fósforo, \%* & - & 0,40 & - \\
Cálcio, \% & & & - \\
\hline
\end{tabular}

*Efeito linear e/ou quadrático $(\mathrm{P}<0,01)$.

digestibilidade de cálcio, que é dependente da fonte utilizada, da atividade fitásica presente, entre outros fatores (Jongbloed et al., 1999).

O resultado de 0,31\% de fósforo digestível está acima das exigências estabelecidas em alguns estudos, como o de Jongbloed \& Everts (1992) e de O’Quinn et al. (1997), que estimaram a exigência de fósforo em 0,23 e 0,21\%, respectivamente. No entanto, esses pesquisadores utilizaram metodologias diferentes para avaliar a exigência de fósforo digestível, sendo que os resultados podem ser influenciados pelas metodologias.

Trabalhos mais recentes compilados nas tabelas FEDNA (Blas et al., 2006) de exigência de fósforo digestível aparente para suínos entre 15 e 50 kg citam valores entre 0,27 e 0,31\% como os mais indicados para máximo desempenho. Por outro lado, Ruan et al. (2007) recomendam o nível de 0,34\% de fósforo digestível como o mais adequado para o desempenho e parâmetros sanguíneos (fósforo no soro) para suínos em crescimento.

\section{Conclusões}

A exigência de fósforo digestível para maximizar o desempenho de suínos alimentados com dietas à base de milho e farelo de soja é de 0,31\%, que corresponde ao consumo de 5,87 g fósforo digestível/dia.

\section{Agradecimentos}

À Serrana Nutrição Animal, pelas análises realizadas nos parâmetros ósseos.

\section{Referências}

ALEBRANTE, L.; DONZELE, J.L.; OLIVEIRA, R.F.M. et al. Available phosphorus levels in diets for pigs with high genetic potential for lean meat deposition kept in thermoneutral environment from 15 to $30 \mathrm{~kg}$. Revista Brasileira de Zootecnia, v.40, p.323-330, 2011.

BLAS, C.; GASA, J.; MATEOS, G.G. Necesidades nutricionales para gado porcino. Normas FEDNA. Madrid, Outubro de 2006, 55p.
BOHLKE, R.A.; THALER, R.C.; STEIN, H.H. Calcium, phosphorus and amino acid digestibility in low-phytate corn, normal corn, and soybean meal by growing pigs. Journal of Animal Science, v.83, p.2396- 2403, 2005

BÜNZEN, S. Digestibilidade do fósforo de alimentos e exigência de fósforo digestível de aves e suínos. 2010. 114f. Tese (Doutorado em Zootecnia) - Universidade Federal de Viçosa, Viçosa, MG.

BÜNZEN, S.; ROSTAGNO, H.S.; LOPES, D.C. et al. Digestibilidade do fósforo de alimentos de origem vegetal determinada em suínos em crescimento e terminação. Revista Brasileira de Zootecnia, v.37, p.1236-1242, 2008.

FAN, M.Z.; ARCHBOLD, T.; SAUER, W.C. et al. Novel methodology allows simultaneous measurement of true phosphorus digestibility and the gastrointestinal endogenous phosphorus outputs in studies with pigs. Journal of Nutrition, v.131, p.2388-2396, 2001.

FERREIRA, D.F. Sistema de análise estatística para dados balanceados (SISVAR). Lavras: Universidade Federal de Lavras/DEX, 2000. Sofware

GOMES, P.C.; RUNHO, R.C.; D’AGOSTINI, P. et al. Exigência de fósforo disponível para frangos de corte machos e fêmeas de 22 a 42 e de 43 a 53 dias de idade. Revista Brasileira de Zootecnia, v.33, p.1734-1746, 2004 (supl. 1).

HASTAD, C.W.; DRITZ, S.S.; TOKACH, M.D. et al. Phosphorus requirements of growing-finishing pigs reared in a commercial environment. Journal of Animal Science, v.82, p.2945-2952, 2004.

JONGBLOED, A.W.; EVERTS, H. Apparent digestible phosphorus in the feeding of pigs in relation to availability, requirement and environment. 2. The requirement of digestible phosphorus for piglets, growing-finishing pigs and breeding sows. Netherlands Journal of Agriculture Science, v.40, p.123-136, 1992.

JONGBLOED, A.W.; KEMME, P.A. Apparent digestible phosphorus in the feeding of pigs in relation to availability, requirement and environment. Digestible phosphorus in feedstuffs from plant and animal origin. Netherlands Journal of Agriculture Science, v.38, p.567-75, 1990.

JONGBLOED, A.W.; EVERTS, H.; KEMME, P.A. et al. Quantification of absorbability of macroelements. In: A quantitative biology of the pig. Wallingford: $C A B$ International, 1999. p.275-298.

KeTAREN, P.P.; BATTERHAM, E.S.; White, E. Phosphorus studies in pig. 1. Available phosphorus requirements of grower/ finisher pigs. British Journal of Nutrition, v.70, p.249-268, 1993.

O’QUINN, P.R.; KNABE, D.A.; GREGG, E.J. Digestible phosphorus needs of terminal cross growing finishing pigs. Journal of Animal Science, v.75, p.1308-1318, 1997.

PETERSEN, G.I.; STEIN, H.H. A novel procedure for measuring endogenous phosphorus losses and true phosphorus digestibility by growing pigs. Journal of Animal Science, v.82 (Suppl. 1), p.254. (Abstr.), 2004.

ROSTAGNO, H.S.; ALBINO, L.F.T.; DONZELE, J.L. et al. Tabelas brasileiras para aves e suínos: composição de alimentos e exigências nutricionais. 2.ed. Viçosa, MG: UFV, Departamento de Zootecnia, 2005. 186p. 
RUAN, Z.; ZHANG, Y.G.; YIN, Y.L. et al. Dietary requirement of true digestible phosphorus and total calcium for growing pigs. Asian-Australasian Journal of Animal Sciences, v.20, p.1236-1242, 2007 (Abstract). Disponível em: <http:// cat.inist.fr/ ?aModele $=$ afficheN\&cpsidt $=18884716>$. Acesso em: 20 jun. 2009.

SARAIVA, A.; DONZELE, J.L.; OLIVEIRA, R.F.M. et al. Available phosphorus levels in diets for swine from 15 to $30 \mathrm{~kg}$ genetically selected for meat deposition. Revista Brasileira de Zootecnia, v.38, p.307-313, 2009.

SHEN, Y.; FAN, M.Z.; AJAKAIYE, A. et al. Use of the regression analysis technique to determine the true phosphorus digestibility and the endogenous phosphorus output associated with corn in growing pigs. Journal of Nutrition, v.132, p.1199-1206, 2002.

SKIBA, F.; CALLU, P.; CASTAING, J. et al. Variabilité intra-matière première de la digestibilité du phosphoredes céréales et du pois chez le porc en croissance. Journées Recherche Porcine, v.36, p.9-16, 2000.
SILVA, J.D.; QUEIROZ, A.C. Análise de alimentos: métodos químicos e biológicos. 3.ed. Viçosa, MG: UFV, 2002. p.235.

STAHLY, T.S. Nutrient needs for high lean pigs. Manitoba agriculture, food and rural initiatives. Disponível em: $<$ http://www.ipic.iastate.edu/reports/00swinereports/asl655.pdf $>$ Acesso em: 21 jun. 2009.

STEIN, H.H.; KADZERE, C.T.; KIM, S.W. et al. Influence of dietary phosphorus concentration on the digestibility of phosphorus in monocalcium phosphate by growing pigs. Journal of Animal Science, v.86, p.1861-1867, 2008.

WIDMER, M.; MCGI.NNIS, R.L.M.; STEIN, H.H. Energy, amino acid, and phosphorus digestibility of high protein distillers dried grain and corn germ fed to growing pigs. Journal of Animal Science, v.85, p.2994-3003, 2007.

WISEMAN, T.G.; MAHAN, D.C.; PETERS, J.C. et al. Tissue weights and body composition of two genetics lines of barrows and gilts from twenty to one hundred twenty-five kilograms of body weight. Journal of Animal Science, v.85, p.1825-1835, 2007. 